\title{
Calagem e as propriedades eletroquímicas e físicas de um latossolo em plantio direto
}

\author{
Liming effect on electrochemical and physical properties of a no-tilled oxisol
}

Falberni de Souza Costa ${ }^{1}$ Cimélio Bayer ${ }^{2}$ Jackson Adriano Albuquerque ${ }^{3}$ Sandra Mara Vieira Fontoura ${ }^{4}$

\section{- NOTA -}

\section{RESUMO}

Neste estudo, avaliou-se (i) o efeito da calagem nas propriedades eletroquímicas e físicas de um Latossolo Bruno há 21 anos em plantio direto (PD), bem como (ii) o efeito do revolvimento do solo para a incorporação de calcário sobre as suas propriedades físicas. A calagem aumentou o potencial elétrico superficial do solo, independente do modo de aplicação de calcário, cujos valores estimados variaram de (-) $90 \mathrm{mV}$ a (-) $118 \mathrm{mV}$. Entretanto, a argila dispersa em água não aumentou, o que pode ter sido devido à sua relação inversa com o carbono orgânico total $(C O T)\left(r^{2}=0,80\right) e$ $\mathrm{Ca}+\mathrm{Mg}\left(r^{2}=0,56\right)$, cujos maiores teores foram verificados na camada superficial do solo. $O$ diâmetro médio geométrico (DMG) dos agregados não foi afetado pela calagem, e teve uma relação positiva com os teores de COT $\left(r^{2}=0,89\right)$ das diferentes camadas de solo. Após 5 anos, não se verificou efeito negativo do revolvimento do solo para a incorporação de calcário sobre o DMG dos agregados e na porosidade deste solo argiloso (690 $\mathrm{g} \mathrm{kg}^{-1}$ argila) e de mineralogia predominantemente gibsítica. Além das condições favoráveis à atividade microbiana, maiores teores de COT e de Ca e $\mathrm{Mg}$ na camada superficial de solos em PD contribuem para a mitigação do efeito dispersivo da calagem.

Palavras-chave: matéria orgânica, solos de carga variável, calcário, argila dispersa.

\section{ABSTRACT}

In this study there has been an evaluation of (i) the liming effect on some electrochemical and physical properties of a no-tilled Oxisol (Haplohumox) for 21 years, and (ii) the effect of soil disturbance to lime incorporation on soil physical properties. Liming increased the surface electric potential, regardless of lime application method, and the estimated values varied from (-) $90 \mathrm{mV}$ to (-) $118 \mathrm{mV}$. However, the amount of clay dispersed in water was not affected by liming, probably due to its negative relationship with total organic carbon (TOC) and $\mathrm{Ca}+\mathrm{Mg}$ concentrations, with the greatest values occurring in soil surface layers. The liming had no effect on the mean geometric diameter (MGD) of soil aggregates, which was positively related to TOC stock of the different soil layers. After five years, no negative effect of plow and disking to lime application into the soil was observed for MGD and porosity of this clayey $\left(690 \mathrm{~g} \mathrm{~kg}^{-1}\right.$ clay) and gibbsitic oxisol, in comparison to surface liming treatment. In addition to the favorable conditions to microbial activity, the highest TOC and $\mathrm{Ca}+\mathrm{Mg}$ in the surface layers of no-tillage soils contribute to mitigated the effect of liming on clay dispersion.

Key words: soil organic matter, variable charge soils, lime, clay dispersion.

A calagem afeta a eletroquímica de solos tropicais de carga variável. O aumento do potencial elétrico superficial contribui para a dispersão de argila, principalmente, de microagregados. Entretanto, a influência da calagem na dispersão de argila, bem como na estabilidade de macroagregados, deve ser dependente do seu efeito no aumento da quantidade de resíduos vegetais adicionados ao solo, os quais são fonte de $\mathrm{C}$ e energia aos microrganismos e, portanto, podem determinar um aumento da atividade microbiana no solo (CHAN \& HEENAN, 1999).

O manejo do solo também pode afetar o efeito do calcário nas propriedades eletroquímicas e físicas do solo. Especificamente no sistema plantio direto (PD), a aplicação superficial de calcário resulta

\footnotetext{
${ }^{1}$ Engenheiro Agrônomo, MSc, doutorando no Programa de Pós-graduação em Ciência do Solo, Universidade Federal do Rio Grande do Sul (UFRGS), Porto Alegre, RS.

${ }^{2}$ Engenheiro Agrônomo, Doutor, Professor do Departamento de Solos, UFRGS. E-mail: cimelio.bayer@ufrgs.br. Autor para correspondência. ${ }^{3}$ Engenheiro Agrônomo, Doutor, Professor do Departamento de Solos, UDESC, Lages, SC.

${ }^{4}$ Engenheiro Agrônomo, MSc, Pesquisador da FAPA, Fundação Agrária de Pesquisa Agropecuária, Guarapuava, PR.
} 
em uma concentração do produto na superfície do solo. Embora seja provável que as alterações no potencial elétrico superficial na camada superficial do solo ocorram em maior magnitude do que quando o calcário é incorporado ao solo, o seu efeito na dispersão de argila não é conhecido. A condição favorável de umidade e temperatura, os maiores teores de matéria orgânica e o acúmulo dos resíduos vegetais sobre o solo favorecem a maior atividade microbiana, com possíveis reflexos mitigadores do efeito dispersivo da calagem em solos cultivados no sistema PD. O objetivo deste estudo foi avaliar o efeito da calagem nas propriedades eletroquímicas e físicas de um Latossolo Bruno cultivado no sistema PD há 21 anos. Adicionalmente, avaliou-se o efeito do revolvimento do solo em PD para incorporação de calcário sobre algumas propriedades físicas do solo, em comparação à sua aplicação superficial.

O experimento localiza-se em área da Fundação Agrária de Pesquisa Agropecuária (FAPA), em Guarapuava, PR. O experimento foi instalado em 1978 e, originalmente, constava da combinação (inverno-verão) dos sistemas PD, preparo convencional (PC) e escarificação (ESC). Apartir de 1987, introduziu-se, em cada combinação de preparos, três tratamentos de calagem (sem calcário, calcário superficial, e calcário incorporado), em subparcelas $(20 \times 30 \mathrm{~m})$. No ano de 1987, aplicou-se 4,5 tha ${ }^{-1}$ de calcário calcítico e, em 1995, foi realizada uma reaplicação na dose de $3 \mathrm{t} \mathrm{ha}^{-1}$ de calcário dolomítico. Para este estudo, amostras de solo foram coletadas em janeiro de 2000, nas profundidades de 0-5, 5-10e 10-20cm, nos três tratamentos de calagem do sistema
PD-PD. Detalhes da amostragem e condução experimental podem ser obtidos em COSTA(2001).

Nas amostras de solo, determinaram-se os teores de carbono orgânico total (COT), Al, Ca e Mg trocáveis, $\mathrm{pH}$ (em água e em $\mathrm{KCl}$ ), ponto de efeito salino nulo (PESN), argila dispersa em água, macroporosidade, densidade do solo e de partícula (EMBRAPA, 1997), bem como foram calculados os valores de delta $\mathrm{pH}$ ( $\mathrm{pH}$ O$\mathrm{pH} \mathrm{KCl}$ ), porosidade total e microporosidade. O potencial elétrico superficial foi estimado pela equação $\Psi_{\mathrm{o}}=59,1$ (PESN - pH-H $\mathrm{H}_{2} \mathrm{O}$ ) (RAIJ \& PEECH, 1972). A estabilidade de agregados em água foi avaliada conforme KEMPER \& CHEPIL (1965) e representada pelo diâmetro médio geométrico (DMG) dos agregados. Os resultados foram submetidos à análise da variância, sendo as diferenças entre médias avaliadas pelo teste de Tukey em nível de $5 \%$ de probabilidade de erro. A relação entre as variáveis foi avaliada a partir da significância dos coeficientes de determinação de regressões lineares.

A calagem aumentou o $\mathrm{pH}$ e os teores de $\mathrm{Ca}$ e $\mathrm{Mg}$, e diminuiu os teores de Al trocável do solo. Contudo não afetou o PESN, o qual variou de 3,30 a 3,40 , caracterizando o solo como de carga líquida negativa, como evidenciado também pelos valores negativos de $\Delta \mathrm{pH}$ (Tabela 1 ).

A calagem aumentou o $\Psi_{\mathrm{o}}$ estimado de (-) $90 \mathrm{mV}$ para (-) 118mv, o qual foi independente do modo de aplicação de calcário e caracteriza a carga variável deste Latossolo gibsítico (Figura 1a). Apesar do aumento do $\Psi_{\mathrm{o}}$ do solo, não se verificou aumento

Tabela 1 - Carbono orgânico total (COT), Ca, $\mathrm{Mg}$ e Al trocáveis, $\mathrm{pH}$ em água, delta $\mathrm{pH}(\Delta \mathrm{pH})$, diâmetro médio geométrico (DMG), porosidade total, macro e microporosidade, em um Latossolo Bruno cultivado em plantio direto por 21 anos, sem calcário, com calcário superficial sem incorporação e calcário incorporado.

\begin{tabular}{|c|c|c|c|c|c|c|c|c|c|c|}
\hline \multirow{2}{*}{ Calcário } & \multirow{2}{*}{ COT } & \multirow{2}{*}{$\mathrm{Ca}$} & \multirow{2}{*}{$\mathrm{Mg}$} & \multirow{2}{*}{$\mathrm{Al}$} & \multirow{2}{*}{$\mathrm{PH} \mathrm{H}_{2} \mathrm{O}$} & \multirow{2}{*}{$\Delta \mathrm{pH}$} & \multirow{2}{*}{ DMG } & \multicolumn{3}{|c|}{ Porosidade } \\
\hline & & & & & & & & Total & Macro & Micro \\
\hline & $\mathrm{g} \mathrm{kg}^{-1}$ & & & $\mathrm{cmol}_{\mathrm{c}} \mathrm{kg}^{-1}$ & & & $\mathrm{~mm}$ & & $\mathrm{~m}^{3} \mathrm{~m}^{-3}$ & \\
\hline & & & & & & $0-5 \mathrm{~cm}$ & & & & \\
\hline Sem & $46 \mathrm{~ns}$ & $3,1 \mathrm{~b}$ & $0,8 \mathrm{~b}$ & $1,2 \mathrm{a}$ & $4,9 \mathrm{~b}$ & $-0,4 \mathrm{~ns}$ & $3,7 \mathrm{~ns}$ & $0,60 \mathrm{~ns}$ & $0,15 \mathrm{~ns}$ & $0,45 \mathrm{~ns}$ \\
\hline Superficial & 47 & $6,0 \mathrm{a}$ & $1,5 \mathrm{a}$ & $0,3 \mathrm{~b}$ & $5,4 \mathrm{a}$ & $-0,5$ & 3,5 & 0,62 & 0,16 & 0,46 \\
\hline \multirow[t]{2}{*}{ Incorporado } & 49 & $4,1 \mathrm{ab}$ & $1,1 \mathrm{ab}$ & $0,7 \mathrm{ab}$ & $5,2 \mathrm{a}$ & $-0,4$ & 4,2 & 0,61 & 0,16 & 0,45 \\
\hline & & & & & & $5-10 \mathrm{~cm}$ & & & & \\
\hline Sem & $36 \mathrm{~ns}$ & $1,5 \mathrm{~b}$ & $0,3 \mathrm{~b}$ & $1,9 \mathrm{a}$ & $4,8 \mathrm{~b}$ & $-0,6 \mathrm{~ns}$ & $2,8 \mathrm{~ns}$ & $0,61 \mathrm{~ns}$ & $0,16 \mathrm{~ns}$ & $0,45 \mathrm{~ns}$ \\
\hline Superficial & 38 & $3,8 \mathrm{a}$ & $0,7 \mathrm{a}$ & $1,0 \mathrm{~b}$ & $5,2 \mathrm{a}$ & $-0,6$ & 2,5 & 0,63 & 0,15 & 0,48 \\
\hline \multirow[t]{2}{*}{ Incorporado } & 41 & $3,2 \mathrm{a}$ & $0,6 \mathrm{a}$ & $0,9 \mathrm{~b}$ & $5,2 \mathrm{a}$ & $-0,6$ & 2,9 & 0,61 & 0,14 & 0,47 \\
\hline & & & & & & $10-20 \mathrm{~cm}$ & & & & \\
\hline Sem & $33 \mathrm{~ns}$ & $1,4 \mathrm{~b}$ & $0,3 \mathrm{~b}$ & $1,5 \mathrm{a}$ & $5,0 \mathrm{a}$ & $-0,6 \mathrm{~ns}$ & $1,6 \mathrm{~ns}$ & $0,61 \mathrm{~ns}$ & $0,15 \mathrm{~ns}$ & $0,46 \mathrm{~ns}$ \\
\hline Superficial & 34 & $3,2 \mathrm{a}$ & $0,6 \mathrm{a}$ & $1,2 \mathrm{~b}$ & $5,2 \mathrm{a}$ & $-0,7$ & 1,7 & 0,65 & 0,15 & 0,50 \\
\hline Incorporado & 32 & $3,1 \mathrm{a}$ & $0,6 \mathrm{a}$ & $0,9 \mathrm{~b}$ & $5,3 \mathrm{a}$ & $-0,7$ & 2,1 & 0,65 & 0,15 & 0,50 \\
\hline
\end{tabular}

Médias seguidas por letras iguais, na coluna, não diferem pelo teste de Tukey ao nível de 5\% de probabilidade de erro; ns=não significativo. 





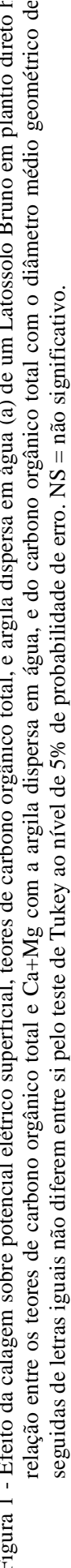

Ciência Rural, v. 34, n. 1, jan-fev, 2004. 
na argila dispersa em água (Figura 1a), ao contrário do que foi observado por CASTRO \& LOGAN (1991) e ALBUQUERQUE et al. (2000) em latossolos do Paraná e Santa Catarina, respectivamente. A calagem também não teve efeito no DMG de agregados, o qual foi relacionado aos teores de COT do solo (Figura 1b).

$O$ não efeito da calagem na dispersão de argila neste solo em PD pode ser devido aos maiores teores de COT (Figura 1a) e de Ca e Mg (Tabela 1) na camada superficial do solo. Na figura $1 b$, verifica-se a relação inversa entre os teores de COT e a argila dispersa em água, a qual pode ser resultante do efeito da matéria orgânica na estabilidade de microagregados, ou pela sua influência positiva na atividade microbiana. Além da matéria orgânica, a atividade microbiana pode aumentar em decorrência da calagem pelo aumento do pH e pela adição de $\mathrm{Ca}$ e $\mathrm{Mg}$, e pela maior adição de resíduos culturais ao solo, cuja magnitude do efeito é potencializada pelas condições mais favoráveis de umidade e temperatura do solo em PD. Por sua vez, o aumento da concentração de $\mathrm{Ca}$ e $\mathrm{Mg}$ atua na floculação das partículas de argila carregadas negativamente, através do mecanismo de ponte de cátions (Figura 1b).

O revolvimento do solo para incorporação do calcário não afetou a estabilidade de agregados e a porosidade do solo (Tabela 1), o que pode estar associado à alta estabilidade estrutural deste solo argiloso $\left(629 \mathrm{~g} \mathrm{~kg}^{-1}\right)$ gibsítico. Importante ressaltar que o solo foi amostrado cinco anos após a última calagem, podendo ter ocorrido uma recuperação das propriedades físicas do solo durante esse período, conforme verificado por REINERT (1998) e CASTRO \&LOGAN (1991).

Associado à maior atividade microbiana, os maiores teores de matéria orgânica e cátions divalentes ( $\mathrm{Ca}$ e $\mathrm{Mg}$ ) atuam na minimização do efeito dispersivo da calagem em solos de carga variável em PD. Esses resultados são importantes quanto à conservação do solo, pois a menor dispersão de argila e maior estabilidade de agregados são fundamentais à maior qualidade física do solo, especificamente no que se refere à infiltração de água e, portanto, para minimizar os riscos de erosão em solos tropicais e subtropicais.

\section{REFERÊNCIAS BIBLIOGRÁFICAS}

ALBUQUERQUE, J.A. et al. Propriedades físicas e eletroquímicas de um Latossolo Bruno afetadas pela calagem. R Bras Ci Solo, v.24, p.295-300, 2000.

CASTRO FILHO, C.; LOGAN, T.J. Liming effects on the stability and erodibility of some Brazilian Oxisols. Soil Sci Soc Am J, v.55, p.1407-1413, 1991.

CHAN, K.Y.; HEENAN, D.P. Lime-induced loss of soil organic carbon and effect on aggregate stability. Soil Sci Soc Am J, v.63, p.1841-11844, 1999.

COSTA, F.S. Propriedades físicas e produtividade de culturas de um Latossolo Bruno sob sistemas de manejo do solo em experimento de longa duração. 2001. $98 \mathrm{f}$. Dissertação (Mestrado em Ciência do Solo) - Pós-graduação em Ciência do Solo. Universidade do Estado de Santa Catarina.

EMBRAPA-EMPRESA BRASILEIRA DE PESQUISA AGROPECUÁRIA. Centro Nacional de Pesquisa de Solos. Manual de métodos de análise de solo. 2. ed. rev. atual. Rio de Janeiro, 1997. 212p.

KEMPER, W.D.; CHEPIL, W.S. Size distribution of aggregates. In: BLACK, C.A.; EVANS, D.D.; WHITE, J.L. (Eds). Methods fo soil analysis. Madison : American Society of Agronomy, 1965. p.499-510. (Agronomy, Monogr., 9).

RAIJ, B.Van,; PEECH, M. Electrochemical properties of some Oxisols and Alfisols of the tropics. Proc Soil Sci Soc Am, v.36, p.587-593, 1972.

REINERT, D.J. Recuperação de solos em sistemas agropastoris. In: DIAS, L.D.; MELLO, J.W.V. (Eds). Recuperação de áreas degradadas. Viçosa : UFV, Sociedade Brasileira de Recuperação de Áreas Degradadas, 1998. p.163176. 